\title{
A High Granularity Timing Detector for the ATLAS detector Phase-II upgrade
}

\author{
H. Imam on behalf of the ATLAS HGTD Collaboration * \\ HASSAN II University - Faculty of Sciences Ain Chock \\ Casablana, Morocco \\ hajar.imam@cern.ch
}

\begin{abstract}
To boost the performance of the Large Hadron Collider and increase the potential of discoveries after 2027, the High Luminosity LHC project has been announced, with the aim of increasing the luminosity by a factor of $\mathbf{1 0}$ above the design value of the LHC. This will lead to an increase of the pileup interactions and will negatively impact the reconstruction of objects in the ATLAS detector, as well as its triggering performance. In particular at the forward region, where the inner detector has a lower momentum resolution and the electromagnetic calorimeter has a coarser granularity. Therefore, a high granularity timing detector has been proposed to mitigate the pile-up effect; complementing the inner tracker and providing a luminosity measurement, it will be located in front of the LAr end-cap calorimeter.

The HGTD will cover the pseudo-rapidity region between 2.4 and 4.0, with two layers of double-sided silicon sensors that will provide a time resolution of 30 ps per track. Each readout cell has a cross-section of $1.3 \mathrm{~mm} \times 1.3 \mathrm{~mm}$ ensuring a high granular detector with 3.7 million channels. To achieve the required high signal-to-noise ratio and provide sufficient gain, Low Gain Avalanche Detector technology was chosen.

The general specifications and requirements of LGAD will be outlined, as well as the technical design and status of the project. The ongoing $R \& D$ effort to study the sensors, readout ASIC, and other components, supported by laboratory and test beam results will also be presented, in addition to some physics and performance results.
\end{abstract}

Index Terms-HL-LHC, ATLAS, HGTD, LGAD, Si sensors, Timing detectors

\section{INTRODUCTION}

The expected increase in particle flux in the high-luminosity phase of the LHC (HL-LHC) [1], with an instantaneous value of up to $\mathrm{L}=7.5 \times 10^{34} \mathrm{~cm}^{-2} \mathrm{~s}^{-1}$, will have a significant impact on the pile-up. The performance for electrons and photons, as well as for jets and missing transverse energy, will be severely degraded in the forward regions of the detector. Therefore, the upgrade of the inner tracker (ITk) [2] will provide a better position resolution and an extended pseudo-rapidity to $|\eta|=$ $4.0(|\eta|<2.5$ up to now ), but even so, this resolution will not meet the requirements of the forward regions. Thus, the time information can help to further reduce the pile-up effect in this regions. For example, by using only ITk, the presence of multiple tracks in close spatial proximity can lead to ambiguities in the track/vertex association. HGTD [3]

* Copyright 2021 CERN for the benefit of the ATLAS Collaboration. Reproduction of this article or parts of it is allowed as specified in the CCBY-4.0 license. time information will effectively reduce the vertex density for a particular track, allowing the hard scattering vertex to be separated effectively from the surrounding pile-up vertices. The expected high levels of radiation in this area require that both the front-end electronics and the detector sensors withstand a $1 \mathrm{MeV}$ equivalent neutron fluence of up to $2.5 \times 10^{15} \mathrm{n}_{e q} \mathrm{~cm}^{-2}$ and a total ionizing dose of $2 \mathrm{MGy}$. The HGTD would reconstruct the time of a minimum ionizing particle (MIP) with a time range of about $30 \mathrm{ps}$ at the beginning of HL-LHC operations to 50 ps toward the end. Due to the necessity of an accurate timing measurement and high resistance to radiation, the sensor choice for HGTD was Low Gain Avalanche Detector (LGAD) [4] pads. Therefore, this project will play a key role in improving the performance of the ATLAS [5] detector in the forward region.

\section{HGTD REQUIREMENTS AND LAYOUT}

\section{A. Overview \& requirements}

In order to guarantee the performance required to meet the high luminosity expected in the HL-LHC phase (200 simultaneous proton-proton interaction within the same bunch crossing interval $(<\mu>=200))$, a part of the ATLAS detector needs to be upgraded, where the electromagnetic calorimeter [6] has a higher granularity and the resolution of the inner tracker degrades. Therefore, it is proposed that the HGTD detector, which will provide high granularity and timing information, be located in the region of the gap between the end-cap calorimeter and the barrel. Each HGTD endcap is an integration of a hermetic vessel, two double-sided instrumented layers, and two moderators placed inside and outside the hermetic vessel. The modules with sensors and on-board electronics are mounted on the front and back of a common cooling disk. Each disk of this latter is physically separated into two circular half disks. The HGTD will cover the pseudo-rapidity range between $2.4<|\eta|<4.0$, complementing the internal tracker by providing high-precision temporal measurements for charged particles, and improving the reconstruction performance of physical objects. Figure 1 shows the position of the HGTD detector.

Based on limitations of space and the expected high level of radiation at HGTD position, a silicon based time sensor technology is chosen to be utilized, with configurable thin array sensors. These demands are fulfilled by the use of $1.3 \mathrm{~mm} \times 1.3 \mathrm{~mm} \times 50 \mu \mathrm{m}$ (length $\times$ width $\times$ thickness) 


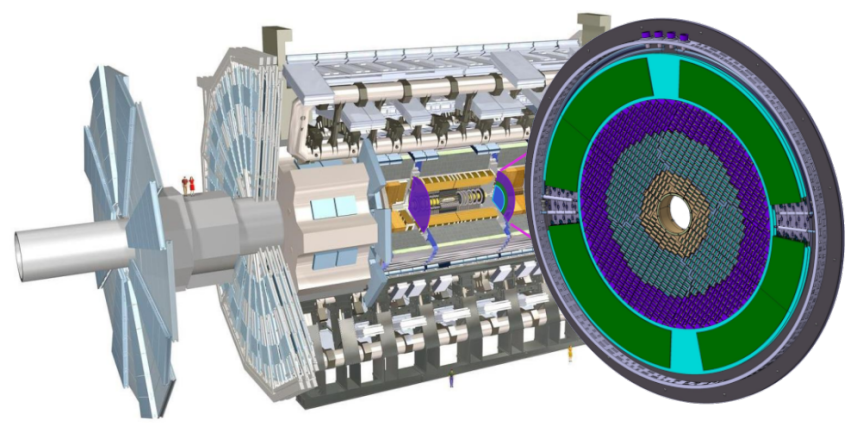

Figure 1: Position of the HGTD within the ATLAS Detector [3].

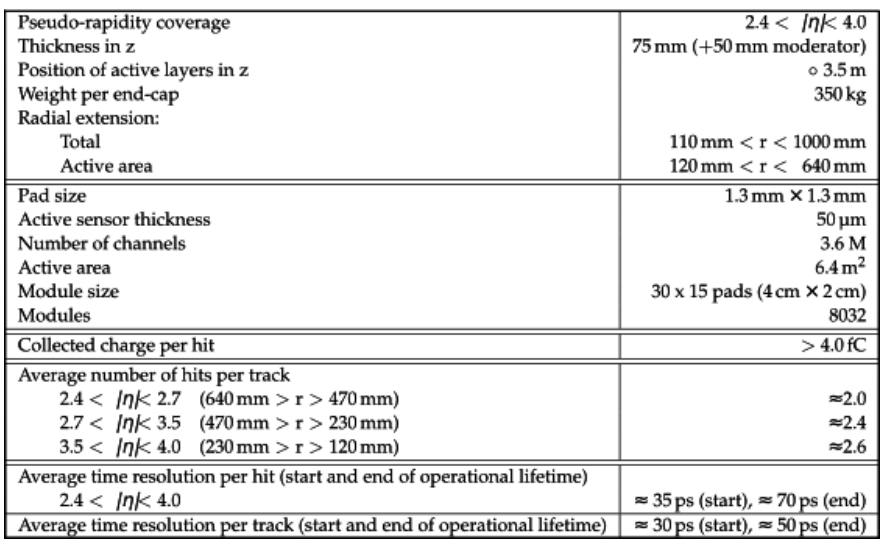

Table I: Main parameters of HGTD [3].

LGAD pads. These sizes ensure less than $10 \%$ occupancy at high pile-up densities, limited inter-pad space and low detector capacitance, which is important for time resolution. For the readout, a custom ALTIROC low-noise ASIC [7], [8] is developed and bumped to the sensors to meet the requirements for timing resolution and resistance to radiation. The ASIC will also provide functionality to count the number of hits registered in the sensor and transmit it at $40 \mathrm{MHz}$ to allow unbiased, bunch-by-bunch measurements of the luminosity and the implementation of a minimum-bias trigger. A time resolution of $35 \mathrm{ps}$ at the start of the HL-LHC (Run 4) is required, followed by a time resolution of $70 \mathrm{ps}$ at the end of the HL-LHC operation period. Table I summarizes the main parameters of the HGTD.

\section{B. Layout}

The objective of the detector design is to ensure the optimal timing resolution to effectively remove the impact of the pileup in the forward region. For this reason, each layer of the HGTD is double-sided, and each side is rotated in opposite directions by $15^{\circ}$. On a common cooling disk, modules with on-detector electronics and sensors are installed on both sides, front and back. The modules are arranged in an overlapping manner, with $20 \%$ overlap for the radius of the disk $r>470$ $\mathrm{mm}, 54 \%$ for $\mathrm{r} \in[230,470] \mathrm{mm}$ and $70 \%$ for $\mathrm{r}<230 \mathrm{~mm}$. The choice of the optimal overlap for each ring was based on a full simulation, in order to achieve the required timing resolution via the average number of simulated hits, given the expected time resolution of the pads.

\section{Radiation hardness}

The radiation hardness of the sensors and electronics is one of the most important parameters of the HGTD. To achieve sufficient performance of the sensors and ASICs, the detector layout has been designed considering a replacement scenario during the HL-LHC. Through an intensive R\&D campaign, a minimum charge of $4 \mathrm{fC}$ is required, this can be achieved up to a radiation damage of $2.5 \times 10^{15} \mathrm{n}_{e q} \mathrm{~cm}^{2}$ and total ionising dose (TID) about 2.0 MGy. As a result, the sensors and electronics within the lowest-radius $(\mathrm{r}<230 \mathrm{~mm})$ will be replaced after each $1000 \mathrm{fb}^{-1}$ and the sensors and ASICs within $230 \mathrm{~mm}<\mathrm{r}<470 \mathrm{~mm}$ should be replaced at half of the data-taking $\left(2000 \mathrm{fb}^{-1}\right)$ during the HL-LHC program. In the inner ring the total $\mathrm{Si} 1 \mathrm{MeV} \mathrm{n}_{e q}$ fluence has a similar proportion of charged particles and neutrons, whereas in the outer and central rings, the effect is dominated by neutrons. To account for uncertainties in the simulation, a total safety factor of 1.5 is applied for the sensors which are most sensitive to the fluence of the particles, and for the electronics which is more sensitive to TID, 2.25 as a factor. The maximum fluence and total ionising dose as a function of the radial position including the replacement of the rings can be found in Figure 2.

\section{Module components}

The hybrid module is made of two parts: two LGAD sensors and two ASICs represent the bare module, and the flexible printed circuit board (flex cables). The flex is made of two pieces, a small flex board permanently glued to the bare module and a long flex tail whose length, of up to about $60 \mathrm{~cm}$, depends on the module position in the detector. The sensors and the ASICs are connected through a flip-chip bump bonding process called hybridization. All connections between the ASIC and the peripheral electronics are routed through the flex cable. The bare module is glued on the back side of the sensor to the flex module small piece, and all the signals are wire bonded between the ASIC and the flex cable and for the high voltage between the sensor and the flex. The size of the bare modules is around $2 \times 4 \mathrm{~cm}^{2}$, the pads size is $1.3 \times$ $1.3 \mathrm{~mm}^{2}$. Each sensor is connected to one ASIC, reading a matrix of 225 channels. A view of the HGTD hybrid module is shown in the Figure 3.

\section{SENSORS AND READOUT ELECTRONICS}

\section{A. LGAD sensors}

LGADs are n-on-p type of silicon detectors with an additional p-layer highly doped below the $n$-p junction to provide a high-field which causes an internal amplification, as illustrated in the top plot of Figure 4. Once a charged particle passes through the detector, an initial current is generated by the electron and the hole drift in the silicon. When electrons reached the amplification region, new electron-hole pairs are created and the holes drift towards the $\mathrm{p}^{+}$area and generate a significant current. This amplification of charge is called 

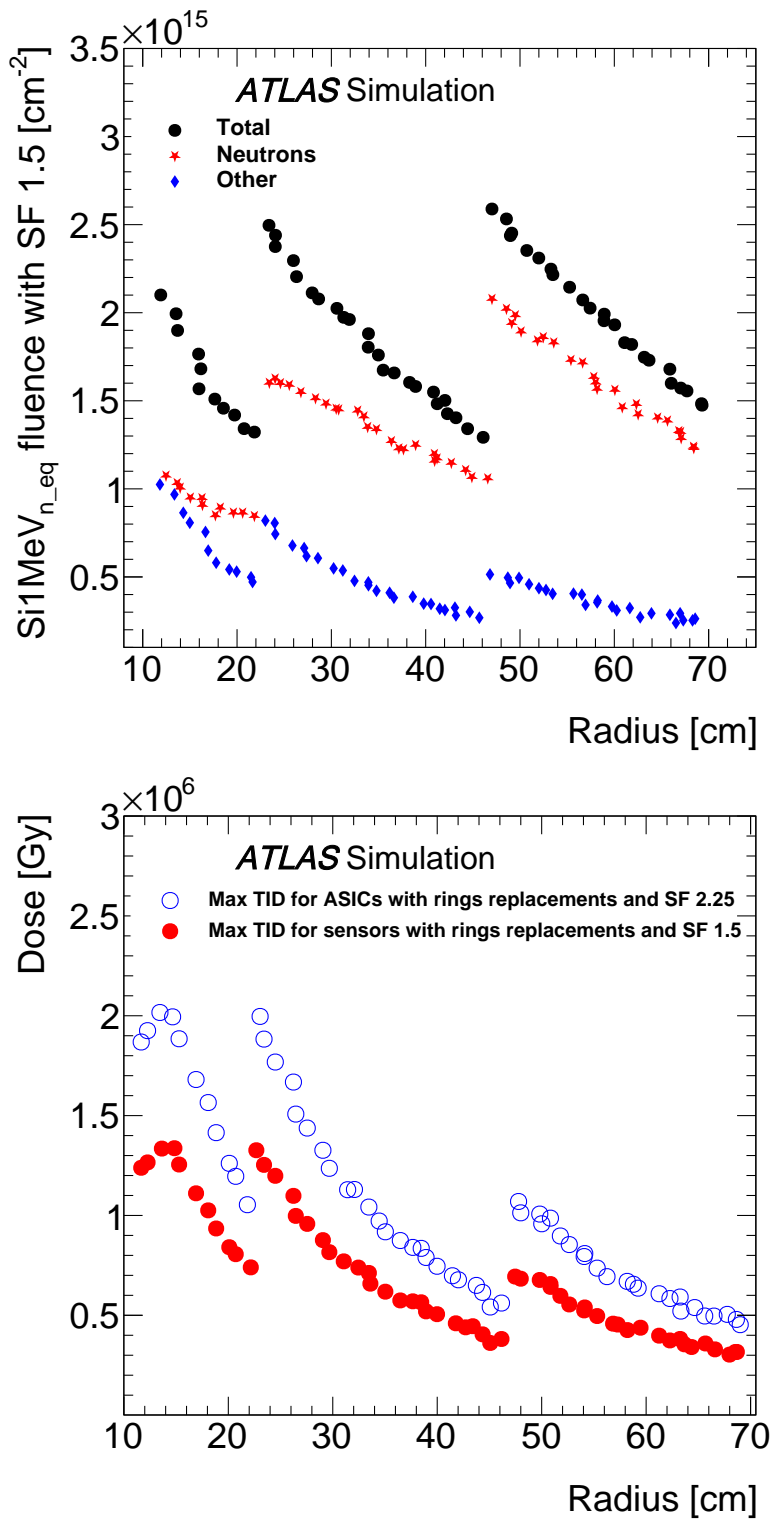

Figure 2: Expected Si $1 \mathrm{MeV} \mathrm{n}_{e q}$ radiation levels in HGTD, using Fluka simulations, as a function of the radius considering a replacement of the inner ring every $1000 \mathrm{fb}^{-1}$ and the middle ring replaced at $2000 \mathrm{fb}^{-1}$ [3].

the LGAD gain. This gain, which is much higher than in a regular diode, is the main component to achieve an accurate time resolution of the deposited energy of the MIPs. Expected values of current for various levels of irradiation (hence various gains) are presented in the bottom of Figure 4. For higher gain, the rise time is approximately 500 ps and the duration of the signal is about $1 \mathrm{~ns}$. As the neutron irradiation fluence increases, the charge is lower and the rise time and signal duration are shorter. The HGTD LGADs were initially designed by the Centro Nacional de Microelectrnica (CNM) in Barcelona [4] and developed during the last 5 years within the CERN-RD50 community [9] including collaboration with two other LGAD vendors: Hamamatsu Photonics (HPK, Japan) and Fondazione Bruno Kessler (FBK, Italy).

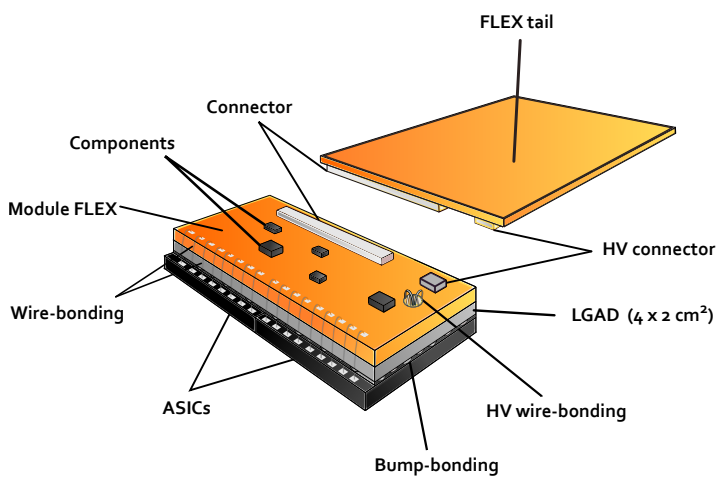

*not to scale

Figure 3: View of an HGTD hybrid module equipped with its read-out flex cable tail [3].

Three major effects determine the time resolution: time walk from amplitude variations, jitter from electronic noise and Landau fluctuations from charge deposition non-uniformities along the particle path. Time walk and noise jitter depend on the type of readout electronics chosen. Both depend inversely on the signal slope (voltage slope at the output of the amplifier) $\mathrm{dV} / \mathrm{dt}$ :

$$
\begin{gathered}
\sigma_{\text {TimeW alk }}=\left[\frac{V_{t h}}{\frac{S}{t_{\text {rise }}}}\right]_{R M S} \propto\left[\frac{N}{\frac{d V}{d t}}\right]_{R M S^{\prime}}, \\
\sigma_{\text {Jitter }}=\frac{N}{\frac{d V}{d t}} \simeq \frac{t_{\text {rise }}}{\frac{S}{N}},
\end{gathered}
$$

where $S$ refers to the signal, which is proportional to the gain, $\mathrm{V}_{t h}$ to the threshold voltage, $\mathrm{t}_{\text {rise }}$ to the rise time and $\mathrm{N}$ to the noise. The third effect, referred to as Landau fluctuation, is due to the non-uniform charge deposition along the particle path leading to time-of-arrival fluctuations. It is a contribution depending on the thickness of the sensor (thin is beneficial) and the setting of the threshold. Adding the three contributions in quadrature yields the overall time resolution. After time walk correction, the noise jitter is the dominating contribution for low $\mathrm{S} / \mathrm{N}$ and the Landau term takes over for high $\mathrm{S} / \mathrm{N}$.

\section{B. Electronics readout}

Considering the expected TID radiation levels and the low jitter required, $130 \mathrm{~nm}$ TSMC CMOS technology was chosen. The overall architecture of the ASIC, called ALTIROC, is similar to ASICs developed for pixel detectors, but with a significantly reduced number of channels and a very different single-pixel front-end optimized for time measurement. Figure 5 shows the general architecture with an array of 225 channels organized in columns for readout and with common digital electronics at the bottom.

The analog front-end electronics of each channel is the most critical element to achieve low jitter. The sensor signal is amplified using a voltage preamplifier. Given the non-negligible duration of the LGAD signal (about $1 \mathrm{~ns}$ ), a preamplifier with a bandwidth of about $1 \mathrm{GHz}$ is sufficient. The preamplifier 


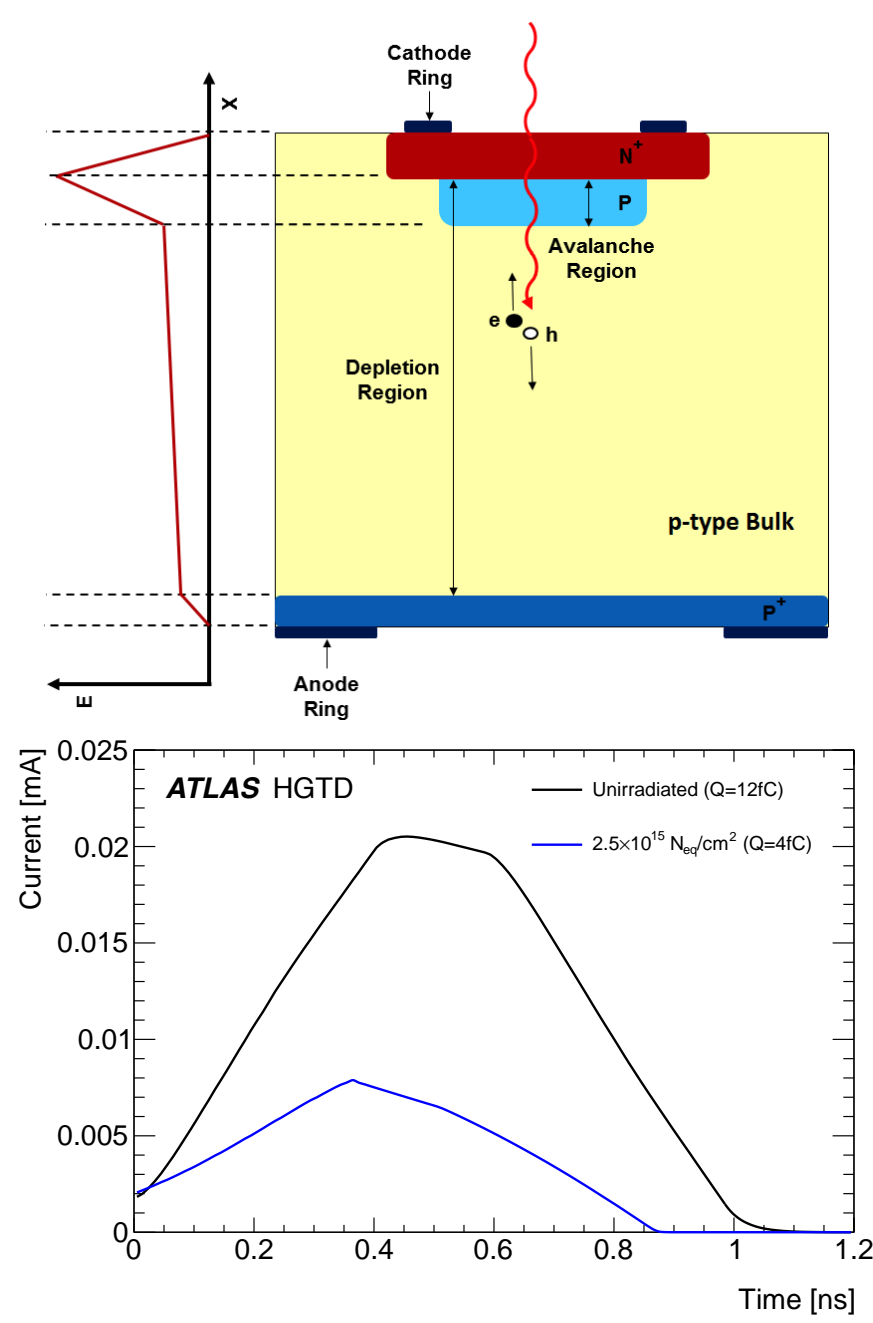

Figure 4: View of the cross section of an LGAD(top), and simulated signal current in LGADs at start and after full integrated neutron fluence(bottom) [3].

is followed by a fast discriminator. The leading edge of the output (Time Of Arrival, or TOA) provides the start of a Time to Digital Converter (TDC) using a Vernier delay line configuration. The stop is given by the clock. This start-stop structure minimizes the power dissipation in case of absence of hits. The quantization step is $20 \mathrm{ps}$, which does not contribute significantly to the expected temporal resolution. TOA measurements are restricted to a $2.5 \mathrm{~ns}$ window centered on the bunch crossing. The expected timing dispersion of the hits has an RMS value of $300 \mathrm{ps}$, so that such a window contains all the hits of the collisions if centered to an accuracy of about 100 ps with a phase shifter. The falling edge of the discriminator output provides the stop for a second TDC (which also uses the leading edge as a start), with a quantisation step of 40 ps, to measure the time over threshold (TOT), which is used as an estimate of the signal amplitude. The TOT information is used offline to correct the TOA for the time walk effect. After correction, the residual variations are well below \pm 10 ps. The digital Front End is used to store the time data up to the reception of a trigger and buffers the data in order to be read by the End Of Column cells. This buffer is needed

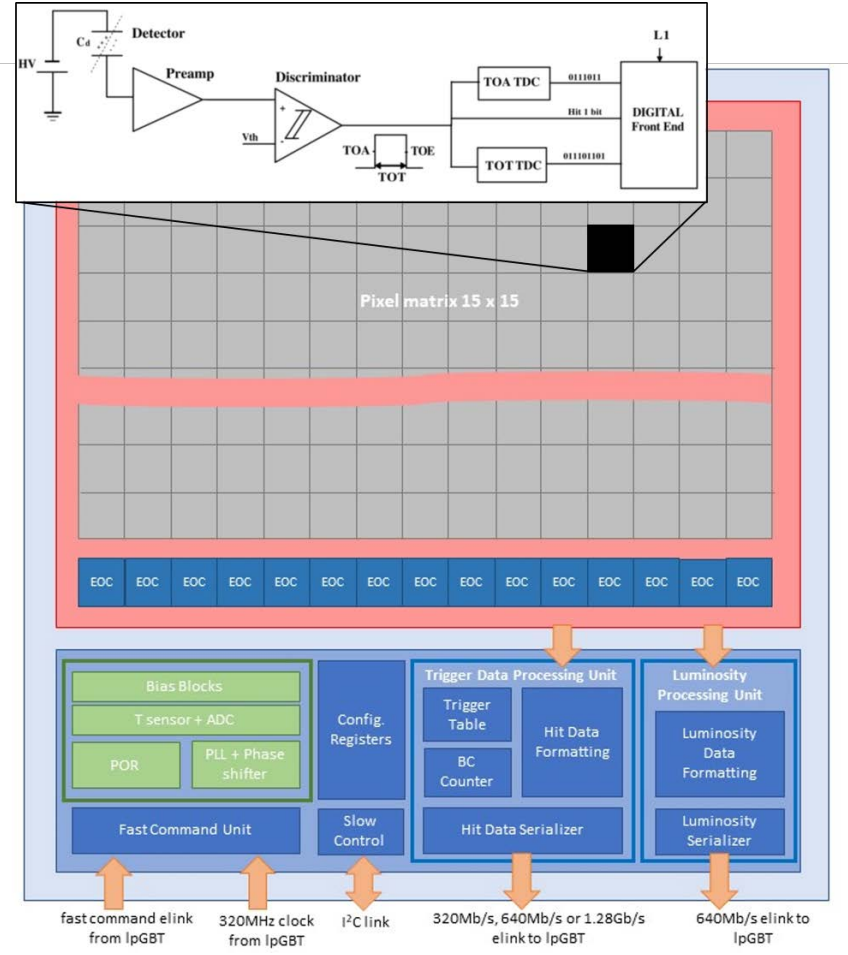

Figure 5: Global architecture of the ALTIROC ASIC. The schematic of one Front End electronics channel is displayed on top of the channels matrix, with the preamplifier followed by a discriminator, two TDCs, and a digital front end block. The ASIC common digital part is shown in the bottom [3].

to cope with event to event fluctuations in the number of hits and random arrivals of the triggers.

Two iterations of this chip have been produced and tested so far: the first, ALTIROC0, integrated four pads in a $2 \times$ 2 array, with the analog part of the single-channel readout: the preamplifier and the discriminator. The second iteration, ALTIROC1, consists of a $5 \times 5$ pad matrix, in which the digital components have been added to the single-channel readout. The next major ASIC iterations, ALTIROC2, will integrate all the functionality of the final ASIC and will have its final size, and the ALTIROC3 which will be the radiation hard version of the ALTIROC2.

\section{SENSORS MEASUREMENTS AT LABORATORY}

Dynamic properties of LGADs, like timing resolution, charge collected, and gain were determined in laboratory using $\beta$ particles from $\mathrm{Sr}^{90}$ source, in response to ionizing particles. Measurements were carried out at $-30{ }^{\circ} \mathrm{C}$ excluding pre-rad measurements which was carried out at $20^{\circ} \mathrm{C}$. Time resolution as function of charge collected for neutron irradiated LGADs from various manufacturers (HPK, FBK) with active thickness of $50 \mathrm{~m}$ is displayed in Figure 6. The error bar is typically about 3 ps. Red line shows that under these circumstances, a time resolution better than $70 \mathrm{ps}$ is achieved at $4 \mathrm{fC}$. Figure 7 illustrates charge collected as function of bias voltage across varying fluences for HPK-3.2 (top) and at maximal fluence for all manufacturers (bottom). Horizontal lines indicate the 


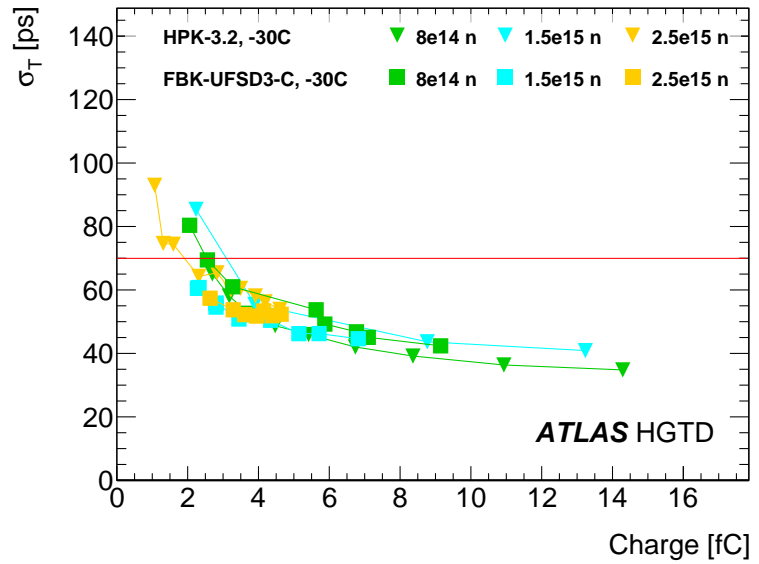

Figure 6: Time resolution as a function of the collected charge for neutron irradiated LGADs from different producers (HPK, FBK) [3].

lower limit of HGTD charge of $4 \mathrm{fC}$ at all fluences. Filled markers show the neutron irradiation (n), empty markers show the proton irradiation at CYRIC (pCy).

These results prove that under irradiation, the expected decline in charge efficiency may be attenuated by increasing the bias voltage ( up to $750 \mathrm{~V}$ of operating voltage) and operating at a low temperature $\left(-30{ }^{\circ} \mathrm{C}\right)$. So a charge of $4 \mathrm{fC}$ can be reach up to a fluence of $3 \times 10^{15} \mathrm{n}_{e q} / \mathrm{cm}^{2}$, providing a time resolution smaller than 70 ps per hit. Furthermore, it is remarkable that the performance of sensors from all manufacturers is quit similar, even if before irradiation the optimal operating bias voltage might be different because the doping profile is different. With a minimal charge of about 4 $\mathrm{fC}$ and a discriminator threshold of about $2 \mathrm{fC}$, a hit efficiency of at least $95 \%$ is expected. For the largest fluence, the boron doping in the gain region has been mostly inactivated and half of the remaining reduced gain is supplied by the bulk diode, due only to the large high bias voltage applied. The time resolution in this domain is fully dominated by the electronics jitter, thus dominated by the ASIC performance at low charge.

\section{TEST BEAM CAMPAIGNS}

To investigate the pre- and post-irradiation performances of the LGAD, as well as the HGTD modules, sensors have been exposed to charged particles beams. The HGTD community has performed the beam test for over fifteen time periods during 2016-2020 at CERN's SPS H6 beamline [11] with 40 to $120 \mathrm{GeV}$ pions, at SLAC with $15 \mathrm{GeV}$ electrons, at Fermilab with $120 \mathrm{GeV}$ protons, and at DESY with $5 \mathrm{GeV}$ electrons [12], [13]. In the next sections, the experimental setup used by the DESY laboratory as a model, and the results of the test beam for the various labs will be presented.

\section{A. Experimental setup}

A telescope type EUDET [14], which is based on mapping MIMOSA pixel planes was used to deliver the position dependent measures. Additional reference plane of pixels was
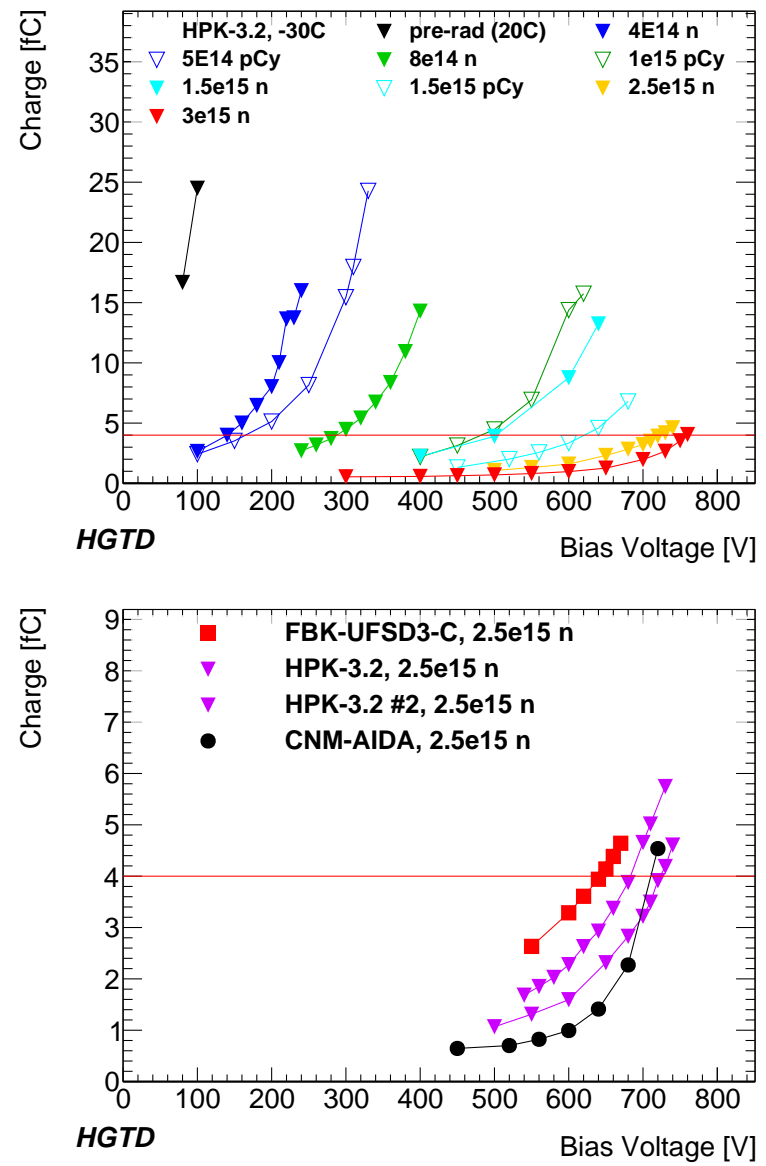

Figure 7: Collected charge as a function of bias voltage for different fluences for HPK-3.2 (top) and at maximum fluence for all vendors (bottom) [10].

also employed for the triggering, utilizing the FE-I4 readout chip [15]. Trigger output signals were merged into the Trigger Logic Unit (TLU), whose output was utilized by the telescope and then linked to the oscilloscope, assuring a perfect matching between events registered by the telescope and the oscilloscope(s). Additionally, quartz bars emitting Cherenkov light connected to silicon photomultipliers (SiPM) have been used as a time reference. The installation was assembled on a plate-type base attached to remote-controlled stage motors which move in both vertical and horizontal directions which are orthogonal to beam direction with micrometer precision. The polystyrene box which covers the tested devices was used to provide both light tightness and temperature stabilization at around $25{ }^{\circ} \mathrm{C}$ using a system of water cooling. To avoid humidity condensing that could harm the sensors, a nitrogen outlet available locally was included in the box.

\section{B. Results}

The charge collected as function of the bias voltage for various sensors fabricated by CNM from the W4 wafer produced in the 10478 run, doped with boron is shown in Figure 8. Measurements were performed at $-32{ }^{\circ} \mathrm{C}$ for sensors irradiated by neutrons (with $6 \times 10^{14} \mathrm{n}_{e q} \mathrm{~cm}^{-2}$ and $1 \times 10^{14} \mathrm{n}_{e q} \mathrm{~cm}^{-2}$ ) or by 


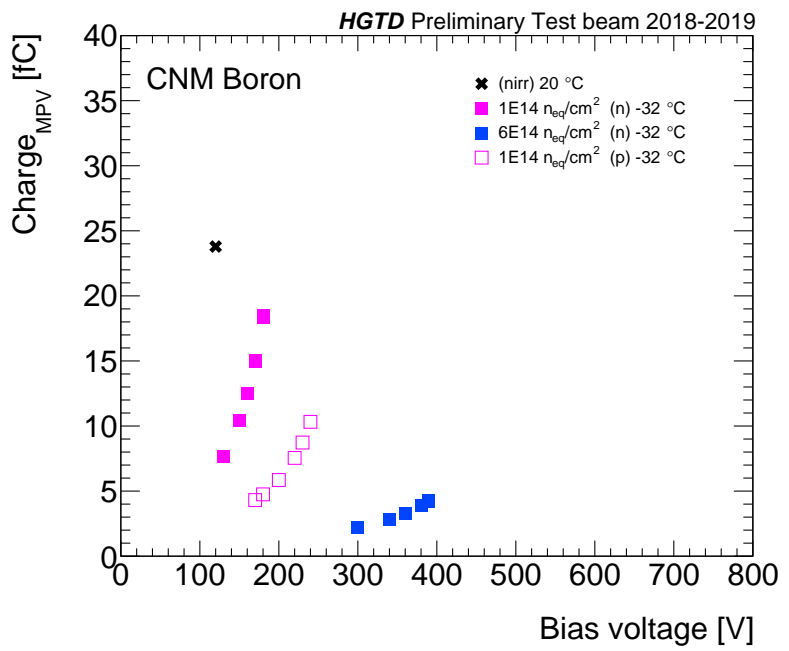

Figure 8: Collected charge as a function of the the bias voltage for different single pad sensors built by CNM [10].

protons ( blank markers) with $1 \times 10^{14} \mathrm{n}_{e q} \mathrm{~cm}^{-2}$ and $20^{\circ} \mathrm{C}$ for the non-irradiated sensors. Fluences are given with a precision of $10 \%$. At every event, the collected waveform charge is computed as the signal area integral. The distribution of the charge is fitted with the Landau-Gauss convolved function, and the charge collected determined as the most probable value from the fitted function. After irradiation of this sensor to $6 \times 10^{14} \mathrm{n}_{e q} \mathrm{~cm}^{-2}$, it achieves $4 \mathrm{fC}$ that is required for best ALTIROC performance, and more precisely $4.2 \mathrm{fC}$ for $390 \mathrm{~V}$.

Constant Fraction Discriminator (CFD) was selected to provide optimal time resolution. Using the CFD algorithm, for every event in a series, the TOA waveform is approximated as the time over which a given threshold is crossed. To identify this threshold, we employ the CFD fraction of the amplitude of the signal, determined by utilizing the dominating time resolution factor. For unirradiated sensors, Landau is the dominant effect, whereas for irradiated sensors, it is noise. To provide a reference time signal, an unirradiated LGAD was used. The time resolution was computed by fitting the time difference distribution between times at a CFD $=50 \%$ for the device under test (DUT) and CFD $=20 \%$ for the non-irradiated sensor. As shown in Figure 9, the boron-doped HPK-3.2 sensors irradiated at $1.5 \times 10^{15} \mathrm{n}_{e q} \mathrm{~cm}^{-2}$ reach the HGTD requirement of time with $36 \mathrm{ps}$, at $600 \mathrm{~V}$ for a 22.8 fC collected charge.

The hit reconstruction efficiency is determined by calculating the ratio of the number of traks that trigger the sensor response (with a collected charge greater than $2 \mathrm{fC}$ ) to the total number of reconstructed traks passing through the DUT at a given position. The efficiency as function of bias voltage for various sensors built by the CNM from the Gallium-doped W6 wafer produced in run 10924 is shown on Figure 10 (top). Sensors have been irradiated by protons or neutrons at varying fluences. Efficiency is computed for a threshold charge of $2 \mathrm{fC}$ corresponding to ALTIROC threshold and based on impacts in the central area of the pad ( within $0.5 \times 0.5 \mathrm{~mm}^{2}$ ). For CNM sensors doped with gallium and irradiated with

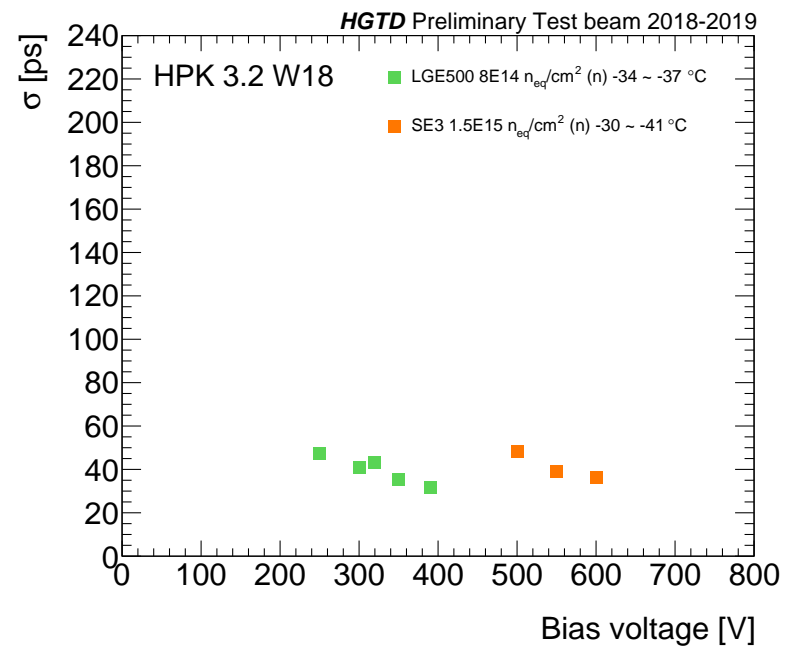

Figure 9: Time resolution as a function of the bias voltage for different single pad sensors built by HPK [10].

$3 \times 10^{15} \mathrm{n}_{e q} \mathrm{~cm}^{-2}$, efficiency is around $99.7 \%$ for $740 \mathrm{~V}$ bias voltage and $5.3 \mathrm{fC}$ as collected charge. The lowest plot in Figure 10 shows the 2D efficiency map of the same sensor for the same bias voltage. This map confirms the fact that the sensor, even after irradiation, stays highly efficient in its center, whereas the borders are less efficient.

An ALTIROC1v2 ASIC bump bonded to a LGAD sensor array (HPK 3.1) was exposed in electron beam tests at DESY in the fall of 2019. The LGADs were operated with a bias voltage of $230 \mathrm{~V}$, resulting in a MIP charge deposit of about $20 \mathrm{fC}$. For an accurate timing reference, a fast Cherenkovlight emitting quartz bar of $6 \times 6 \mathrm{~mm}^{2}$ area transverse to the beam and $20 \mathrm{~mm}$ length along the beam, coupled to a Silicon Photomultiplier (SiPM) is used. The time resolution of this device was measured to be $37.6 \pm 0.7 \mathrm{ps}$. The top plot of Figure 11 shows the TOA variation as a function of the TOT. The range of the TOT is truncated since it was not possible to measure large values of TOT because of a coupling between the busy signal of the TOA TDC and the falling edge of the preamplifier output. This coupling only occurs when this signal is output on the PCB. This TOA busy signal must be output during test beam in order to synchronize the data from ALTIROC and the oscilloscope used to record the Quartz+SiPM system waveforms. This signal will not be needed for the HGTD and is not used for test bench measurements (it is only used for debug purposes). In the next iteration, ALTIROC1v3, the busy signal will be output as a differential signal to solve this problem. Therefore, with ALTIROC1v2, only a range of the TOT can be used in test beam and the top plot of Figure 11 also displays a fit in this restricted range used for the time walk correction.

The bottom plot of Figure 11 shows the time difference between LGAD+ALTIROC and the reference time from the Quartz+SiPM system before and after time walk correction extracted from the fit in the top of Figure 11. The distributions are Gaussian without any tails. The measured time resolution decreases from $58.3 \pm 1.6$ ps to $46.3 \pm 1.4$ ps after time 

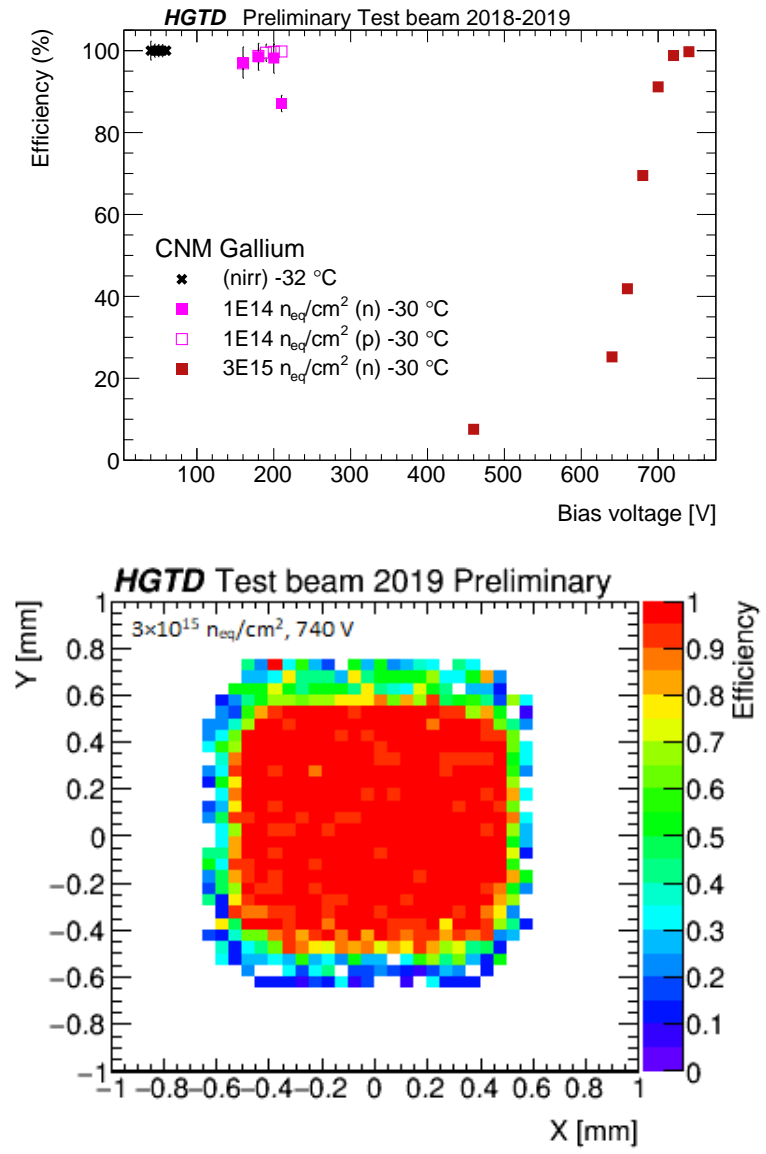

Figure 10: Efficiency as a function of bias voltage for different single pad sensors built by CNM (top), Signal efficiency in a $1 \times 1 \mathrm{~mm}^{2}$ CNM Gallium-doped LGAD irradiated to $3 \mathrm{e} 15$ $\mathrm{n}_{e q} / \mathrm{cm}^{2}$, as a function of the reconstructed position $\mathrm{X}$ and $\mathrm{Y}$ of particles (in mm) (bottom) [10].

walk correction. Substracting the Landau contribution (about $25 \mathrm{ps}$ ), the remaining time resolution is about 39 ps containing contributions from the electronics jitter, TDC and clocks.

\section{HGTD PERFORMANCES AND PHYSICS}

The new capability introduced by the HGTD to provide a vertex $t_{0}$ as well as time information for forward tracks can be exploited to mitigate the impact of pile-up in high-level physics object reconstruction, complementing the ITK in the forward region. Those object-level improvements can then, in turn, increase the physics potential of ATLAS. In this section we will focus on how the HGTD can improve the suppression of forward pile-up jets and the efficiency of forward lepton isolation based on full simulation studies.

\section{A. Suppression of pile-up jets}

Pile-up jets can reduce the precision of Standard Model measurements and the sensitivity to discover new physics. For example, additional jets can increase the number of background events passing a selection, as well as reduce the efficiency of kinematic variables or discriminants to separate signals from backgrounds. Hence, the efficient identification
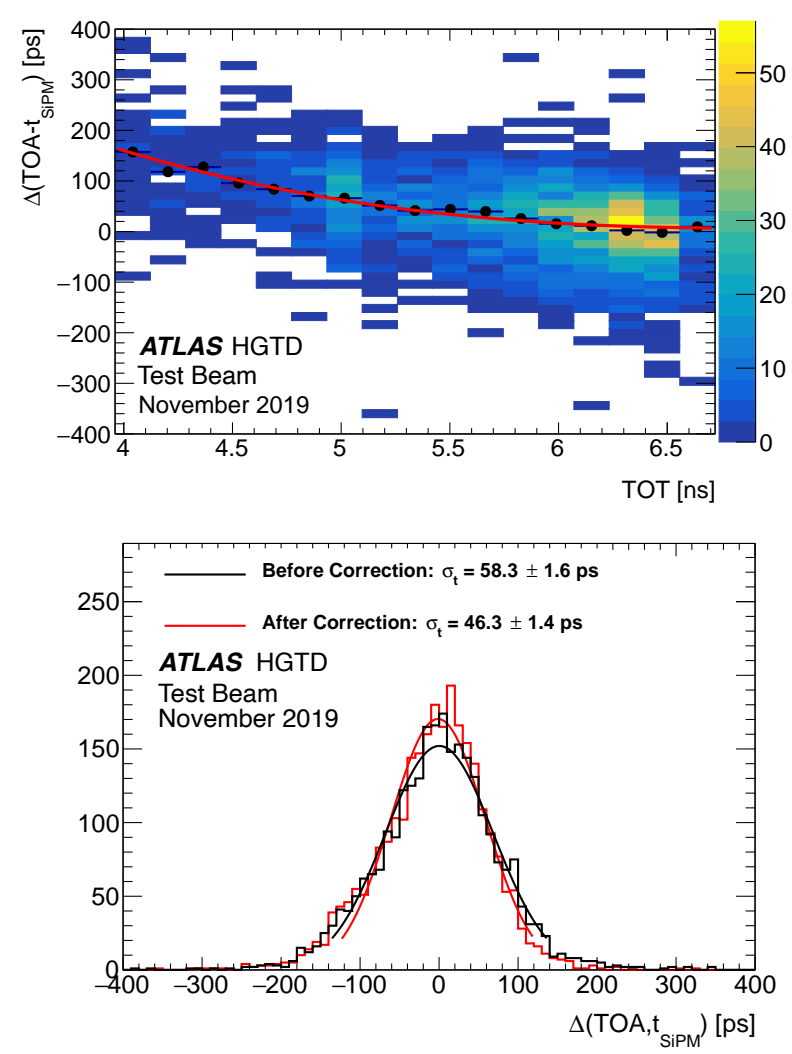

Figure 11: Distribution of the TOA as a function of the TOT (top), Distributions of the time difference between LGAD+ALTIROC and the Quartz+SiPM system before (red) and after (black) time walk correction together with Gaussian fits (bottom) [3].

and rejection of pile-up jets are essential to enhance the physics potential of the HL-LHC. These pile-up jets can be produced as the result of a hard QCD process (QCD jets) from a pile-up interaction, or by random combinations of particles from multiple vertices. At low jet $\mathrm{p}_{\mathrm{T}}$, the latter mechanism is dominant, whereas at high jet $\mathrm{p}_{\mathrm{T}}$, the majority of pile-up jets are QCD jets. Figure 12 shows the pile-up jet rejection as a function of hard-scatter jet efficiency in the $2.4<|\eta|<4.0$ region at the beginning of the lifetime of the detector, $\mathrm{VBF}$ $\mathrm{H}$ to invisible sample, for the ITk-only and combined ITk + HGTD reconstruction, in the top: Jets with $30 \mathrm{GeV}<\mathrm{p}_{\mathrm{T}}<$ $50 \mathrm{GeV}$, and in the bottom: Jets with $\mathrm{p}_{\mathrm{T}}>50 \mathrm{GeV}$.

\section{B. Lepton track isolation}

The ability to assign a time to leptons can be exploited to reduce the impact of pile-up in the case of applying track-isolation criteria to leptons in the forward region. The efficiency of the track-based lepton isolation is defined as the probability that no additional tracks with $\mathrm{p}_{\mathrm{T}}>1 \mathrm{GeV}$ are reconstructed within $\Delta \mathrm{R}=\sqrt{(\Delta \eta)^{2}+(\Delta \phi)^{2}}<0.2$ of the lepton track. In the forward region, the relatively large $\mathrm{z}$ window required to associate tracks to the primary vertex results in increased pile-up track contamination, which consequently degrades the isolation efficiency. The association of a time to the lepton track can be utilized to reject tracks 

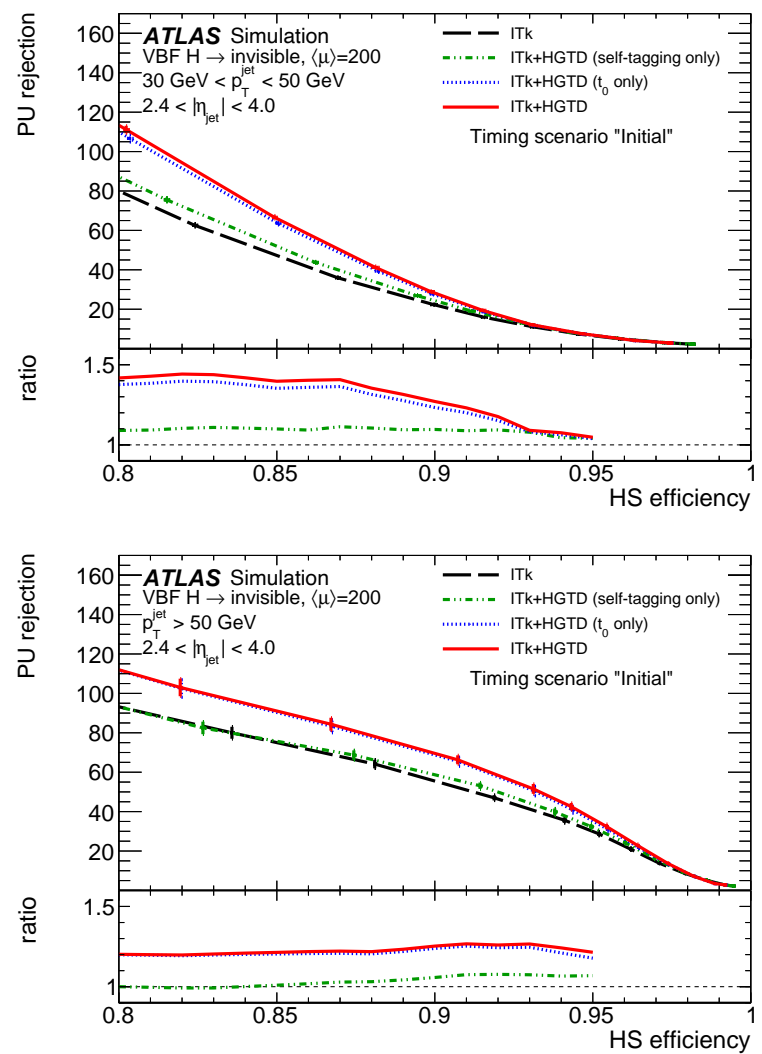

Figure 12: Pile-up jet rejection as a function of hard-scatter jet efficiency,Jets with $30 \mathrm{GeV}<\mathrm{p}_{\mathrm{T}}<50 \mathrm{GeV}$ (top), Jets with $\mathrm{p}_{\mathrm{T}}>50 \mathrm{GeV}$ (bottom) [3].

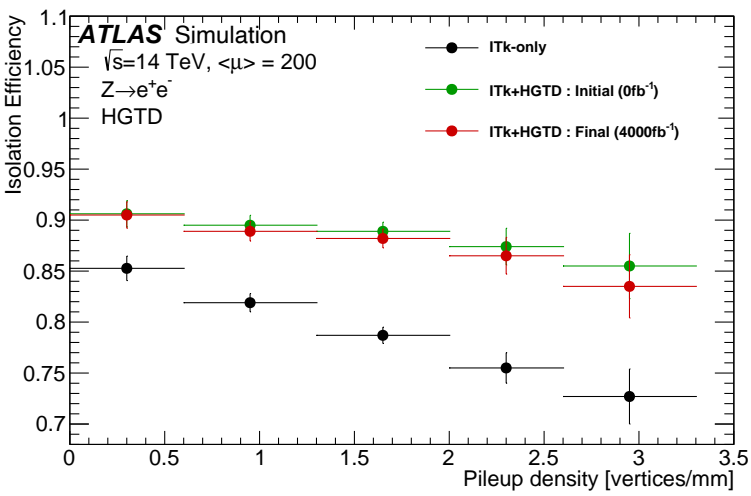

Figure 13: The efficiency for electrons to pass track-isolation criteria as function of the local vertex density [3].

within the isolation cone which come from pile-up interactions spatially close to the hard-scatter vertex. Figure 13 illustrate the efficiency for electrons to pass track-isolation criteria as function of the local vertex density, for the ITk-only and ITk+HGTD scenarios. The isolation efficiency is shown for both the initial time resolution of the detector as well as the final time resolution at the end of lifetime.

\section{CONCLUSION}

As previously stated, the HGTD detector will provide a powerful new tool to mitigate the pile-up effect in the forward region of the ATLAS detector. It is planned to be built with LGAD detector technology, providing a time resolution of $30-50$ ps per track until the end of the HL-LHC lifetime. R\&D studies indicate that LGAD will improve the physics performances of the ATLAS detector in the denser HL-LHC environment. In addition, the development of this technology will open up new applications for LGAD sensors in fields other than particle physics. The ongoing $\mathrm{R} \& \mathrm{D}$ is being conducted by about 150 physicists, engineers, and technicians from 30 ATLAS institutes and 13 countries/funding agencies, which shows the importance of this project. This work was carried out between 2018 and 2020, and further R\&D is still in progress. The detector construction is planned to be achieved by 2026 , followed by installation, integration and comissioning by 2027 .

\section{REFERENCES}

[1] ATLAS Collaboration, High-Luminosity Large Hadron Collider (HLLHC): Technical Design Report V. 0.1, ser. CERN Yellow Reports: Monographs. Geneva: CERN, 2017. [Online]. Available: http: //cds.cern.ch/record/2284929

[2] ATLAS Collaboration, "Technical Design Report for the ATLAS Inner Tracker Pixel Detector," CERN, Geneva, Tech. Rep., Sep 2017. [Online]. Available: https://cds.cern.ch/record/2285585

[3] ATLAS Collaboration, "Technical Design Report: A High-Granularity Timing Detector for the ATLAS Phase-II Upgrade," CERN, Geneva, Tech. Rep., Jun 2020. [Online]. Available: https://cds.cern.ch/record/ 2719855

[4] G. Pellegrini, P. Fernández-Martínez, M. Baselga, C. Fleta, D. Flores, V. Greco, S. Hidalgo, I. Mandić, G. Kramberger, D. Quirion, and M. Ullan, "Technology developments and first measurements of Low Gain Avalanche Detectors (LGAD) for high energy physics applications," Nuclear Instruments and Methods in Physics Research Section A: Accelerators, Spectrometers, Detectors and Associated Equipment, vol. 765, pp. 12-16, 2014. [Online]. Available: https://www.sciencedirect.com/science/article/pii/S0168900214007128

[5] ATLAS Collaboration, "The ATLAS Experiment at the CERN Large Hadron Collider," JINST, vol. 3, p. S08003. 437 p, 2008 , also published by CERN Geneva in 2010. [Online]. Available: https://cds.cern.ch/record/1129811

[6] ATLAS Collaboration, "ATLAS Liquid Argon Calorimeter Phase-I Upgrade: Technical Design Report," Tech. Rep., Sep 2013, final version presented to December 2013 LHCC. [Online]. Available: https://cds.cern.ch/record/1602230

[7] C. Agapopoulou, S. Blin, A. Blot, L. Castillo García, M. Chmeissani, S. C. Di Lorenzo, C. de La Taille, P. Dinaucourt, A. Fallou, J. G. Rodriguez et al., "Performance of a front end prototype asic for picosecond precision time measurements with lgad sensors," Journal of Instrumentation, vol. 15, no. 07, p. P07007, 2020.

[8] C. de la Taille, S. Callier, S. Conforti, P. Dinaucourt, G. MartinChassard, N. Seguin-Moreau, C. Agapopoulou, N. Makovec, L. Serin, and S. Simion, "ALTIROC0, a 20 pico-second time resolution ASIC for the ATLAS High Granularity Timing Detector (HGTD)," PoS, vol. TWEPP-17, p. 006, Sep 2017. [Online]. Available: https://cds.cern.ch/record/2319292

[9] RD50 Collaboration. [Online]. Available: https://rd50.web.cern.ch/rd50

[10] ATLAS HGTD Public Plots. [Online]. Available: https://twiki.cern.ch/ twiki/bin/view/AtlasPublic/HGTDPublicPlots

[11] C. Allaire, J. Benitez, M. Bomben, G. Calderini, M. Carulla, E. Cavallaro, A. Falou, D. Flores, P. Freeman, Z. Galloway, E. Gkougkousis, H. Grabas, S. Grinstein, B. Gruey, S. Guindon, A. Henriques Correia, S. Hidalgo, A. Kastanas, C. Labitan, D. Lacour, J. Lange, F. Lanni, B. Lenzi, Z. Luce, N. Makovec, G. Marchiori, L. Masetti, A. Merlos, F. McKinney-Martinez, I. Nikolic-Audit, G. Pellegrini, R. Polifka, D. Quirion, A. Rummler, H. Sadrozinski, A. Seiden, L. Serin, S. Simion, E. Spencer, S. Trincaz-Duvoid, 
M. Wilder, A. Zatserklyaniy, D. Zerwas, and Y. Zhao, "Beam test measurements of Low Gain Avalanche Detector single pads and arrays for the ATLAS High Granularity Timing Detector," JINST, vol. 13, p. P06017. 28 p, Apr 2018. [Online]. Available: https://cds.cern.ch/record/2313951

[12] R. Diener, J. Dreyling-Eschweiler, H. Ehrlichmann, I. M. Gregor, U. Kötz, U. Krämer, N. Meyners, N. Potylitsina-Kube, A. Schütz, P. Schütze, and M. Stanitzki, "The DESY II test beam facility," Nuclear Instruments and Methods in Physics Research Section A: Accelerators, Spectrometers, Detectors and Associated Equipment, vol. 922, pp. 265-286, 2019. [Online]. Available: https://www.sciencedirect. com/science/article/pii/S0168900218317868

[13] L. Castillo Garcia, "A High-Granularity Timing Detector for the Phase-II upgrade of the ATLAS Calorimeter system: detector concept, description and R\&D and beam test results," CERN, Geneva, Tech. Rep., May 2020. [Online]. Available: https://cds.cern.ch/record/2717972

[14] H. Jansen, S. Spannagel, J. Behr, A. Bulgheroni, G. Claus, E. Corrin, D. Cussans, J. Dreyling-Eschweiler, D. Eckstein, T. Eichhorn et al. "Performance of the eudet-type beam telescopes," EPJ Techniques and Instrumentation, vol. 3, pp. 1-20, 2016. [Online]. Available: https: //link.springer.com/content/pdf/10.1140/epjti/s40485-016-0033-2.pdf

[15] U. Koetz and I. Rubinskiy, "User manual: Atlas fe-i4a pixel module as a trigger plane for the beam telescope," 2013 [Online]. Available: https://telescopes.desy.de/images/0/08/131014_ koetz-rubinsky_FEI4_as_trigger_plane.pdf 